\title{
Modelling of different circular end-of-use scenarios for smartphones
}

\author{
Rainer Pamminger ${ }^{1} \mathbb{D}$. Sebastian Glaser $^{1} \cdot$ Wolfgang Wimmer $^{1}$
}

Received: 21 February 2020 / Accepted: 13 January 2021 / Published online: 2 February 2021

(c) The Author(s) 2021

\begin{abstract}
Purpose Repairing, refurbishing and remanufacturing are three strategies of the Circular Economy (CE), aiming at closing product cycles and maintaining materials and resources in the product cycle as long as possible. This paper analyses the environmental impacts of these three circular end-of-use scenarios (repairing, refurbishing and part remanufacturing) when performed on a common, "non-circular" smartphone. The underlying data used for this paper partly have been result of the Horizon 2020 project sustainablySMART, where circular product concepts have been developed and analysed in detail.

Methods To analyse the environmental impacts of different circular end-of-use scenarios of smartphones, a Life Cycle Assessment (LCA) is performed. For considering the impact of a smartphone's first life (e.g. materials, production), an economic allocation is used.

Since the goal of the study was to better understand the environmental impacts of processing routes that enable multiple life cycles of a product, allocation according to the economic value is applied instead of applying system expansion. As system expansion provides just an aggregated view of the first and second product life cycles and no decision support at the end of the first life regarding the relevant CE strategy can be given. The economic allocation is based on the ratio between the residual market value and the original price from the scenario's input stream of smartphones of the respective end-of-use scenario. To reach comparability of the results, a second-use-time-parameter is defined for each scenario. This parameter takes into account that the second use time reaches only a certain share from the average smart phone use time.

Results This study shows that through all three circular strategies, a reduction in the investigated impact categories-Global Warming Potential (GWP) and Abiotic Depletion Potential (ADP)—can be achieved.

Conclusions The analysed end-of-use scenarios repairing and refurbishing show the highest potential for smartphones in terms of Circular Economy, as most of the environmental impacts can be allocated to the device production, and the impact of additional steps to perform CE-strategies (e.g. collection of discarded phones, refurbishing) is rather low.
\end{abstract}

Keywords Life cycle assessment · Smart mobile devices · Circular Economy $\cdot$ Repair $\cdot$ Reuse $\cdot$ Remanufacture $\cdot$ Recycling $\cdot$ SustainablySMART

\section{Introduction}

Circular Economy (CE) is a concept aiming at maintaining the value of products, materials and resources in the economy for as long as possible, and generating minimum waste through establishing product cycles (European Commission 2015). As such, the CE proposes certain

Communicated by Guido W. Sonnemann.

Rainer Pamminger

rainer.pamminger@tuwien.ac.at; pamminger@ecodesign.at

1 TU Wien, Institute of Engineering Design and Product Development, Research Area ECODESIGN, Vienna, Austria strategies to prolong the lifetime of products after endof-use: reuse, repair, refurbishing, remanufacturing and recycling.

Repairing is an effective way to reduce the environmental impact of smartphones, by restoring the functionality of the product as soon as it breaks down, and by extending the life of the product. The result of repairing old, but functional devices, is that fewer new devices are needed. Many shops offer smartphone repairs to smartphone users, and lately, a "do it yourself" trend is observable, as repair online tutorials, and providers of tools and spare parts such as IFIXIT are becoming popular.

Refurbishing in this context means that discarded smartphones go through a process of refinishing, to serve 
their original functions. Refurbished devices are in trend, as they are an affordable option compared with newly produced smartphones (Mishra 2018). One popular refurbishing company, Refurbed, buys large quantities of used smartphones from companies or telecommunications providers, and runs them through a complete refurbishment process: testing, component replacement if needed, data erasure and cleaning. After this process, smartphones look and work as good as new and include a warranty (Refurbed GmbH 2019).

Remanufacturing valuable electronic components and using them to produce new smartphones is rarely applied today, even though some electronic components such as semiconductors show high potential for remanufacturing due to their high value and environmental impact. The semiconductor technology has a very fast innovation cycle, resulting in a high variation of available semiconductors. The difficulty is then to find appropriate phones for the use of remanufactured semiconductors. Considering the high circularity potential of the remanufacturing strategy and the research attention it has gained, the strategy is taken into account in this analysis nevertheless.

Recycling is not considered in this study, as per definition the full value in the product, and its components are lost in the recycling process. However, the material value is kept, and therefore, recycling presents a considerable end of life option.

\section{Goal and scope}

The goal of this paper is to guide decisions concerning CEstrategies of smartphones after its first life, i.e., when is it advisable to consider multiple product life cycles, and which CE-strategy should be selected from an environmental perspective? What are the environmental impacts of the processes to realize multiple life cycles? How do these impacts differ? As such, the paper includes relevant information particularly for manufacturers or operators, who process discarded smartphone.

The paper assesses each of the selected CE-strategy in a separate end-of-use scenario. In each modelled scenario, the functionality of the smartphone is maintained: through successful repairing, refurbishment, or through a new production, but with remanufactured components (e.g. semiconductors). Although a multi-stage reuse process would be more realistic, e.g. after a damage, the phone is repaired, then it is reused, after that the device is given as a donor, where some parts are reused as spare parts, would be more realistic, the paper assesses the three CE-strategies separately for reasons of simplicity and comparability.

In all scenarios, the strategies apply to common (noncircular) smartphones. Additional hardware design (e.g. built-in additional connectors for modularity of devices) to ease the realization of Circular Economy strategies are therefore not included.

\section{Method}

Life Cycle Assessment (LCA) according to ISO 1404044 is used to assess the environmental performance of the described end-of-use scenarios. Such an LCA includes different steps which are described in detail the following: definition of goal and scope including declared unit (Sect. 3.2), the system boundary (Sect. 3.3), the inventory analysis including the processes needed (Sect. 3.4), the different allocation methods used (Sect. 3.5), the results of the impact assessment (Sect. 4) and the final interpretation and discussion of results (Sect. 5).

Within an LCA often considered full life cycle of a product includes the extraction of raw materials, manufacturing, distribution, use phase and end-of-life phase. If the LCA considers a circular approach, the product (here: smartphone) runs through additional use phases, with further processes to prolong the lifetime, are needed. The conducted LCA includes therefore additional use phases to assess the additional environmental impact, and as such, the LCA shows a higher complexity especially in terms of allocation.

This study follows an attributional approach as in this paper the environmental impact of a product system namely a smartphone, no other product system is considered and the calculation is built up on and highly dependent on LCAs from literature which are also using the attributional approach.

The paper aims at providing information to manufacturers or refurbishers, who are able to control the production process of smartphones, and as such, the use phase and the final end-of-life phase (e.g. disposal of phones: landfill, material recovery and thermal recovery) are not within the scope of this paper. This is also because smartphones are not energy intensive products during their use phase; the major environmental impact of smartphones (about $75 \%$ of the Global Warming Potential (GWP); see Fig. 9) derives from the used materials and resources, as well as the production (Proske et al. 2016a). The environmental impact of discarded smartphones in the end-oflife phase is relatively low, as the positive and negative impacts are roughly balanced out (ibid.). 


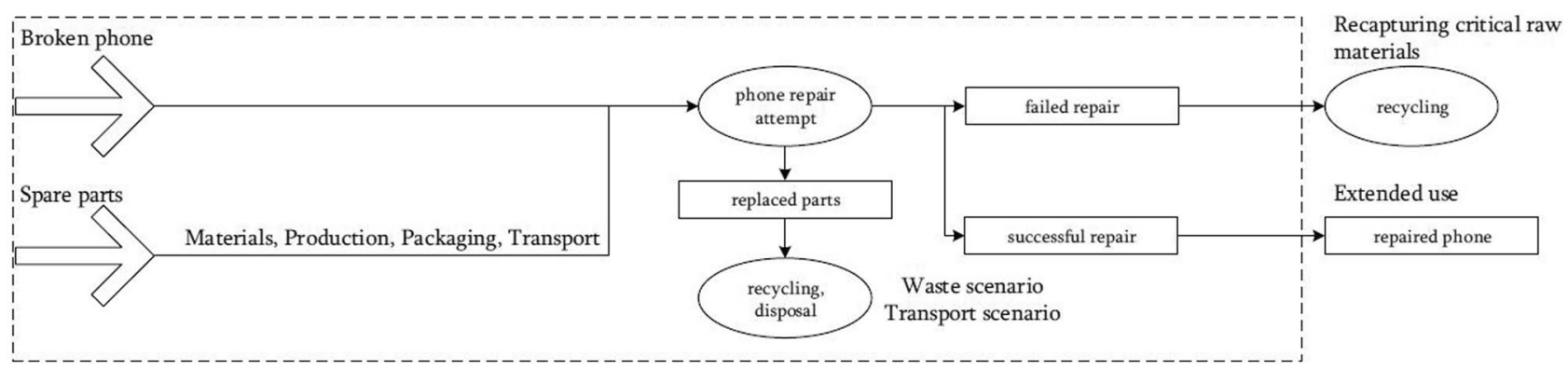

Fig. 1 Product system with elementary flows and product flows, scenario 1, smartphone repair

\subsection{LCA tool and database}

The Life Cycle Assessment has been performed using the software SimaPro v8 (PRé 2020), and background data from the database Ecoinvent v3.5 have been used for the materials, processes, electricity and transport (Ecoinvent 2020). Specific data used for the processes over the life cycle of the product were measured, calculated or assumed as described in Sect. 3.

\subsection{Declared unit}

The declared unit is defined as "one working smartphone for a use time of 2.5 years". The average use time of 2.5 years is chosen according to (Wieser and Tröger 2017), (Proske et al. 2016b). Depending on the applied CE-strategy (repair, refurbishing, remanufacturing), the product's second use time is likely to be less than 2.5 years. Therefore, a seconduse-time-parameter is defined, which scales the actual second use time onto the average use time. In the context of this study, "working smartphone" refers to the technical and functional features of a smartphone implemented by the original manufacturer in the original product.

\subsection{System boundaries}

\subsubsection{Geographical location}

All modelled scenarios refer to the European region. The collection of discarded smartphones, recycling, refurbishing and remanufacturing are assumed to take place in Europe. The reference smartphone and the spare parts are produced in Asia, and shipped to Europe.

\subsubsection{CE-Strategies}

The conducted LCA considered the processes of the three CE-strategies as well as distributions, including the collection of discarded phones, and transports to the sites of repairing, refurbishing or remanufacturing. In the following, the three CE-strategies and the considered processes are described in detail.

\subsubsection{Repair}

In this strategy, broken smartphones are repaired (Fig. 1) by their users or by professional repair shops. The most common reasons of defects have been analysed based on different surveys and studies, from which an average defect variation was derived, as shown in Table 1 (Wieser and Tröger 2017; Statista 2016; Proske et al. 2016a; premium concepts GmbH 2013).

The average defect variation includes the probability of damage type for a broken smartphone (e.g., 37\% of the damaged phones have a broken display). The chosen defect variation in this study focuses on the most common defects: broken display, weak or defect battery and mechanical defects (mainly related to the housing). Water damages are not included. The repair scenario is modelled as follows: Spare parts are produced in Asia and shipped to Europe. Broken parts (according to the defect variation) to be replaced are discarded through a specific waste scenario. A certain percentage of failed repair attempts is also considered.

\subsubsection{Refurbishing}

The second strategy considered is smartphone refurbishing: used smartphones are reconditioned and sold to new users. In the chosen scenario, used phones are first collected and then transported to the recycling site, where they get sort by

Table 1 Assumed defect variation for broken smartphones for smartphone repairing and refurbishing scenario

\begin{tabular}{lc}
\hline Defect & Probability \\
\hline Broken display & $37 \%$ \\
Weak or broken battery & $34 \%$ \\
Mechanical defects & $6.5 \%$ \\
Other defects & 22.5 \\
\hline
\end{tabular}




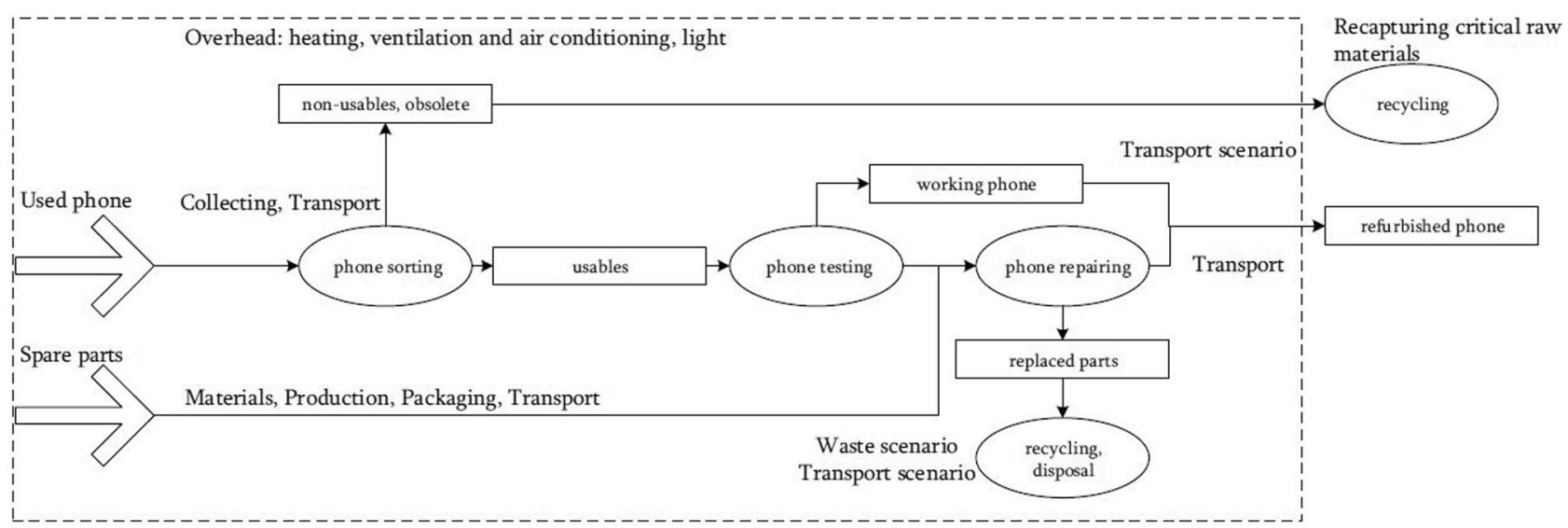

Fig. 2 Product system with elementary flows and product flows, scenario 2, smartphone refurbishment

"refurbishable" phones (Fig. 2), and by non-usable phones, which go through further recycling processes. The selected phones are transported to the refurbishing company, and the average defect variation for replacing broken parts is applied (Table 1). The considered refurbishing process steps are checking the phones condition, charging, testing, replacing components if needed, testing, data erasure and resetting to factory state. Finally, the refurbished phones are distributed to new users for their second life.

\subsubsection{Remanufacturing}

Remanufacturing is a strategy aiming at extracting valuable components from discarded smartphones, and using them for the production of new phones. Due to the high costs of semiconductor components, the further use of integrated circuits, microphones, cameras, etc., are of relevance for the remanufacturing strategy (Pamminger et al. 2019). For the Apple iPhone 6, the integrated circuits are the most expensive components. In terms of the environmental performance, the central processing unit (CPU), Random-access memory (RAM) and flash memory account for $35 \%$ of the GWP of the smartphone production phase (Proske et al. 2016a). As mentioned before, remanufacturing of electronic components such as semiconductors is rarely practiced. However, as this strategy was examined within the sustainablySMART (Ciszewski et al. 2018), a remanufacturing scenario was also modelled and included in the paper.

Figure 3 shows the remanufacturing process: smartphones are collected and transported to the recycler. At the recycling site, the phones are sorted, semi automatically dismantled and de-polluted (removal of battery), and the printed circuit boards (PCB) are extracted. The PCBs are then transported to the remanufacturer. At the remanufacturing site, the relevant parts (semiconductors) are de-soldered, cleaned and remanufactured (Ciszewski et al. 2018). After de-soldering, the PCBs with the remaining parts are transported back to recycler. The remanufactured parts are used for the production of new smartphones.

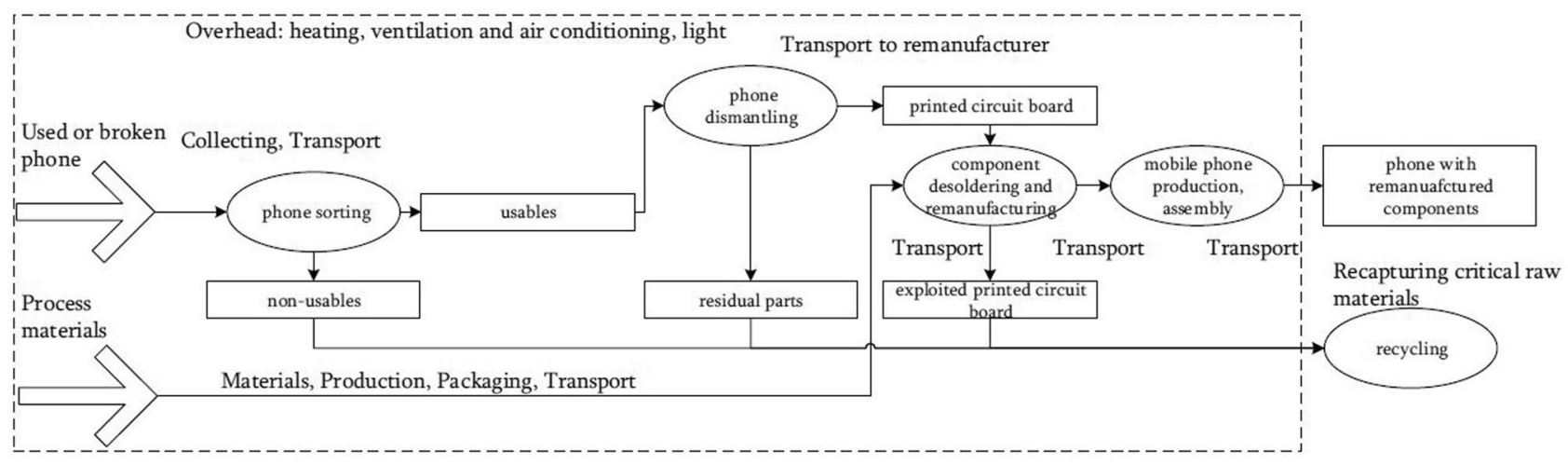

Fig. 3 Product system with elementary flows and product flows, scenario 3, extracting and remanufacturing of semiconductor components for the production of new smartphones 


\subsection{Life cycle inventory}

\subsubsection{Reference smartphone}

A reference smartphone is used in this study for modelling the three end-of-use-scenarios as well as for the linear scenario (same power demand, same functionality, etc.). The reference smartphone represents a common smartphone, with no specific design to ease circular strategies, e.g. the use of additional gold for module connectors, etc. For modelling the reference smartphone, inventory data from previously conducted and published smartphone LCAs (Proske et al. 2016a; Ercan and Kimfalk n. d.; Güvendik 2014) was used. The results were compared and adopted to be in the midrange of the LCA results from literature.

\subsubsection{Processes}

As described, the three end-of-use scenarios partly follow the same distribution, and therefore show similar process steps (Fig. 4). These steps were considered in the research project sustainablySMART. Each process step has been developed and some demonstrators were built. Specific data, like the measurements of an automated disassembly machinery, etc., could be generated from this demonstrators. The individual process steps and their underlying data are described in detail:

(a) Smartphone collecting

For the refurbishing as well as for the remanufacturing scenario, the quality of the input stream of collected phones (the ratio of phones in good condition, and phones in poor condition) is assumed to be 1:4. A transport scenario with a total of $1125-\mathrm{km}$ road and 375-km rail (Proske et al. 2016a) to a recycling site within Europe, where the smartphone sorting and possible dismantling takes place, is chosen.

\section{(b) Smartphone sorting}

According to the input stream, phones of poor quality (unusable or badly damaged phones, etc.) are sorted out. In the modelled scenario, this process step takes place at the recycler. About $1.4 \mathrm{Wh}$ energy per phone of good quality is measured on a prototype of an automated sorting machinery, developed by REFIND Technologies. For lighting, heating, ventilation and air conditioning (HAVAC) at the recycling site, $5.2 \mathrm{Wh}$ per phone was calculated based on the space requirement of the machinery equipment; the process duration and average energy need for a industrial site.

(c) Smartphone dismantling

For the phone dismantling and the PCB extraction, an energy consumption of $17 \mathrm{Wh}$ per phone was measured on a real demonstrator (phone opening, battery and PCB extraction). About $63 \mathrm{Wh}$ per phone for lighting and HVAC was calculated based on the space requirement of the machinery equipment; the process duration and average energy need for a industrial site.

(d) PCB transport to remanufacturing/smartphone transport to refurbishing

For the chosen scenario, the extracted PCBs are transported $800 \mathrm{~km}$ within Europe per lorry, to the remanufacturing or the refurbishing site.

(e) Part de-soldering and remanufacturing

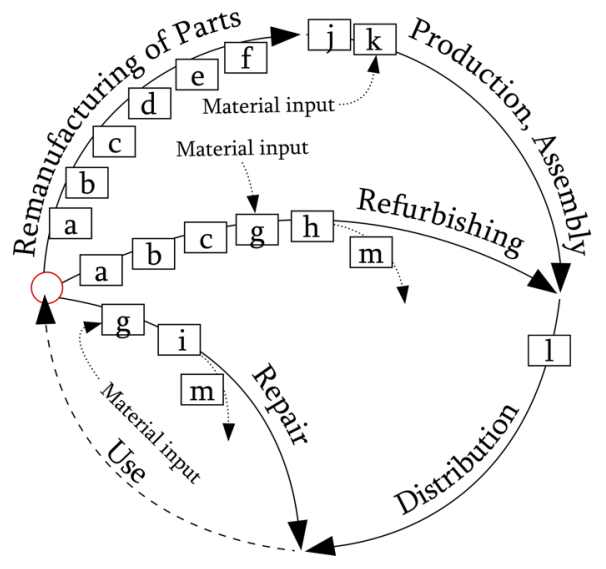

a. Smartphone collecting

b. Smartphone sorting (at recycler)

c. Smartphone dismantling and PCB extraction (at recycler)

d. PCB transport to remanufacturer / Smartphone transport to the refurbisher

e. Part desoldering and remanufacturing (CPU and memories)

f. PCB transport back to recycler

g. Spare parts transport

h. Smartphone refurbishing: testing, repairing, resetting, cleaning

i. Smartphone repair

j. Remanufactured parts transport

k. Smartphone production and assembly

1. Smartphone distribution

m. Replaced parts disposal

Fig. 4 End-of-use scenarios repair, refurbishing and remanufacturing and their process steps 
A thermal stress-minimized de-soldering process, T3E3 technology, as elaborated by Semicon and ITR (Janusz et al. 2016), is used as reference. The energy consumption covering the process steps (PCB drying, flux application, component de-soldering, residual solder removal, flux application, placing new solder balls, soldering the solder balls, cleaning, component drying and component laser marking) was measured to be in the range of $200 \mathrm{Wh}$ per component (Fig. 5), and the material input (solder, flux) was analysed. About $800 \mathrm{Wh}$ are assumed for lighting and HVAC, based on PCB manufacturer data (Kupka 2018). Based on a published study (Janusz et al. 2016), the T3E3 remanufacturing process and its environmental impacts were investigated on five different semiconductor components from digital voice recorders on experimental level: a digital signal processor (DSP), a microcontroller (Fig. 5), a small flash memory, RAM memory and a Rockchip CPU. As the reference smartphone's components (especially the memories) are bigger than the analysed semiconductors, the environmental impact for chip remanufacturing was scaled up according to the following ratio, using the Global Warming Potential as indicator: "GWP of materials and production of new components/GWP of the chip remanufacturing (materials, remanufacturing processes)." The impacts due to transport (smartphone collecting and PCB transport to the remanufacturing site) are independent from the component size and were not scaled up.

\section{(f) PCB transport back to recycler}

After part de-soldering, the exploited PCBs are returned to the recycler for standard recycling, by means of the same transport as described in the previous section d, PCB transport to remanufacturing/Smartphone transport to refurbishing. The recycling of PCB is not in the scope of this study.

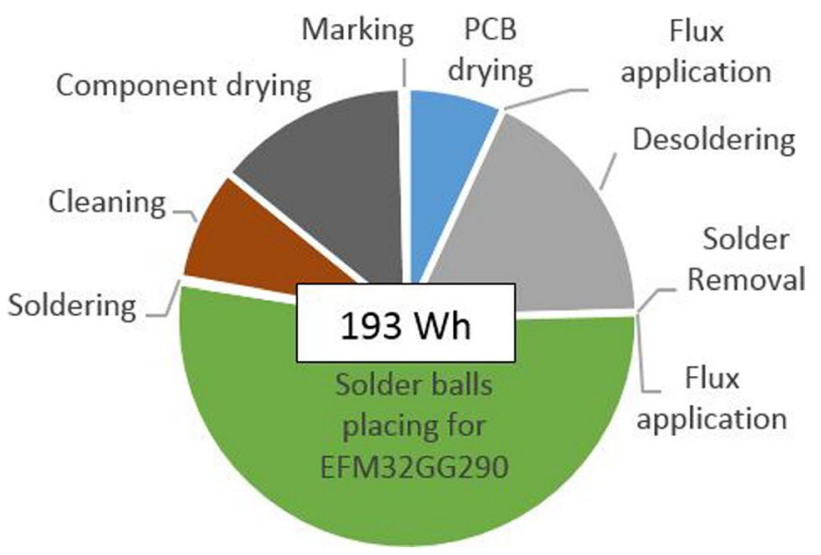

Fig. 5 Energy required for a microcontroller remanufacturing process, T3E3 technology (g) Spare parts transport

Depending on the defect variation in the repair or refurbishing scenario, different spare parts need to be acquired. The production of those spare parts is assumed to take place in Asia, and a transport scenario with $7200-\mathrm{km}$ air freight and 800-km lorry (Ercan 2013) for the repair scenario are chosen. For the refurbishing scenario (transport from the production site of spare parts to the refurbishing site) $7200-\mathrm{km}$ air freight and $200-\mathrm{km}$ lorry for the are chosen.

(h) Smartphone refurbishing: testing, repairing, resetting, cleaning

About $70 \%$ of the phones in use are replaced by customers when still in perfectly functioning condition (Wieser and Tröger 2017), meaning that only $30 \%$ of the discarded phones are defect according to the smartphone defect variation (Table 1), and need components to be replaced. This equals to $11 \%$ of the total smartphone input stream need a display replacement and $10 \%$ a new battery. Compared with the modelled repair scenario, in which only necessary parts are replaced to restore full functionality, customers expect that a purchased, refurbished phone looks and works like a newly manufactured one. Therefore, functional, but worn-out components need to be replaced. Therefore, it is assumed that the e.g. housing of each second smartphone is replaced. Based on Skerlos et al. (2003), about $350 \mathrm{Wh}$ for the refurbishing process steps smartphone are needed. Although the study did cover refurbishing steps for feature phones, the data is assumed to comply with current smartphone refurbishing processes, as the processes are the same (use of compressed air, battery charging test cycles, functionality tests cycles and energy overhead like lighting and HAVAC).

(i) Smartphone repair

As opposed to the refurbishing scenario, the repair scenario only covers the replacement of broken parts. In this study, the three major defects of smartphones are considered: broken display, weak or defect battery and mechanical defects (Table 1). According to Wieser and Tröger (2017), 70\% of the attempted repairs fail (user survey) because the problem cannot be identified or because the phones are beyond repair. Since only services of professional repair shops are considered, a repair success rate of $30 \%$ is very low. Therefore and as small repair shops also claim $90 \%$ success rates, a repair success rate of $75 \%$ was chosen and a sensitivity analysis with reduced rates of 50\% and $33 \%$ was conducted to analyse the impact of this estimation (see chapter 5.2). 
(j) Remanufactured parts transport

Extracted semiconductor parts are transported to Asia, where the smartphone production takes place.

(k) Smartphone production and assembly

The production step is modelled in the same way as for the reference smartphone, with the difference of using remanufactured parts instead of new ones. Therefore, this step is modelled without the materials and production for those new parts.

\section{(1) Smartphone distribution}

After smartphone production and assembly $(k)$ or after smartphone refurbishing $(h)$, the phones have to be distributed to the customer. It was assumed that the refurbishing takes place in Europe (800-km road), and the production of a smartphone with remanufactured parts in Asia (7200-km air freight and 800-km road).

\section{(m) Replaced parts disposal}

Parts replaced during repair or refurbishing are allocated to different disposal scenarios. For the repair scenario, $81 \%$ of the parts are disposed on landfills and 19\% are recycled. For the refurbishing scenario, it was assumed that $17 \%$ are disposed to landfills, and $83 \%$ are recycled (Ercan and Kimfalk n. d.).

\subsection{Allocation}

Since the goal of the study was to better understand the environmental impacts of processing routes that enable multiple life cycles of a product, applying system expansion, where the additional processes are included (preferred acc. to ISO 14040, as allocation is avoided), might not bring sufficient and detailed insights on specific benefits and burdens. System expansion provides an aggregated view of the first and second product life cycles, but the strength of this study does not lie in the aggregation and averaging, instead in demonstrating the merits of each CE-strategy at the product level. Therefore, allocation was needed and allocation based on the economic value was used for different processes. Nevertheless, the paper briefly discusses the results of a possible system expansions approach (Sect. 5). To support decision-making when choosing suitable CE-strategies for discarded smartphones, this paper focuses on the additional processes required to extend a smartphone's life and evaluates the relevant environmental aspects and impacts. A specific allocation approach is taken for the smartphone's parts from the first life, which are e.g. reused in the second life. The corresponding burdens are allocated to the modelled CE-scenarios. The ISO 14044 standard allows to use the change of the economic value as a base for allocation, among other options (change of physical properties or number of subsequent life cycles). The economic value best describes the loss of quality of used or defect smartphones. A smartphone with a long-lasting design, which is easy to repair and with guaranteed (software) updates, will last longer. This quality differences are also depicted on the second hand market, which shows a higher demand for long-lasting devices, and thus higher selling prices for used (and repaired/refurbished) phones (new Fairphone II 399€ vs. refurbished Fairphone II 299€, Fairphone B. V. spring 2019). However, also smartphones that are not known to be long-lasting or easy to repair also show a high customer demand, e.g. premium segment (higher general quality). Higher price ranges for used premium segment smartphones such as the Apple iPhones are clearly observable on sale platforms. It is plausible to assume that the quality of a product's second life is reflected in the market price. For the allocation from the product's first to second life, the study uses the ratio "residual market value, used or broken devices/original price."

\subsubsection{Economic allocation for the smartphones}

To identify the allocation ratio between the products' first and second life, the ratio "residual market value, used or broken devices/original price" from the input stream of smartphones of the end-of-use scenario is calculated.

For smartphone refurbishing as well as for smartphone repair, the premium segment smartphones are of interest, as they have a high resale value (Wieser and Tröger 2017). Hence, selected smartphones introduced to the market from 2014 to 2018 are considered in this study: Apple iPhone 6, 7, 8 and XR and Samsung Galaxy S 6, 7, 8 and 9. For each model, the original price and prices for the devices in different conditions (= residual market values) were researched (Table 2; for Apple iPhone 6), using the sales platform www.rebuy.de.

Table 2 Residual market value (EUR) for Apple iPhone6 in different conditions, Source: www.rebuy.de, 2019

\begin{tabular}{ll}
\hline Apple iPhone 6 & \\
\hline Original price & $€ 699$ \\
Perfect condition & $€ 185.28$ \\
Used (assumed for weak battery) & $€ 59.04$ \\
Used and broken display & $€ 0$ \\
Used (assumed for broken housing) & $€ 59.04$ \\
Perfect condition, but unknown defect & $€ 0$
\end{tabular}


The scenarios analysed in this study (Figs. 1, 2, and 3) are based on different input streams of smartphone qualities. For the repair scenario, $100 \%$ of input stream are broken phones according to defect variation (Table 1), and for the refurbishing scenario, $70 \%$ of the inputs are assumed to be in perfect conditions, whereas $30 \%$ need repair according to the defect variation (Table 1).

For both scenarios (repair, refurbishing) the residual market values (for iPhone6, 7, 8 and XR and Samsung Galaxy $\mathrm{S} 6,7,8$ and 9) considering the defect variation were calculated. In a second step, the allocation ratios (residual market value to original price) were calculated, for each model and brand (Table 3).

allocation ratio $=\frac{\text { residual market value }}{\text { original price }}$

To calculate the average allocation ratio for "one average broken phone" for the repair scenario, the individual market share of the different models have to be considered (assuming an equal probability of a defect-e.g. broken displayfor each phone model).

average allocation ratio $=\sum$ allocation ratio $*$ market share

Generally, the distribution of smartphones in use varies according to the age (Jkielty 2019). Both the newest and older smartphones models have a lower market share, while the majority of customers is using last year's models. According to Jkielty's web traffic data, the individual market share for Apple iPhone is iP6 29\%, iP7 41\%, iP8 16\% and iPX 14\% and for Samsung Galaxy S S6 18\%, S7 43\%, S8 $33 \%$ and S9 7\% (2019). This leads to an allocation ratio for a broken iPhone of $11 \%$ and for a broken Samsung Galaxy phone of 9\%. Considering the market share of Android and iOS devices (e.g. as published on www.statista.com), the average repair allocation adds up to $10 \%$.

For the refurbishing scenario, the average allocation ratio is calculated similarly by using the allocation ratios per model (Table 3 ) and the age distribution of the phones not in use any more. For the age distribution, phone replacement cycle survey data is used. Based on the assumption that the phones are bought in the release year, $25 \%$ of the

Table 3 Ratio residual market value to original price, according the refurbishing- or repair scenario per model (\%)

\begin{tabular}{lllll}
\hline Apple iPhone & iP6 & iP7 & iP8 & iPXR \\
\hline Refurbishing scenario & $19 \%$ & $31 \%$ & $44 \%$ & $46 \%$ \\
Repair scenario & $3 \%$ & $12 \%$ & $16 \%$ & $17 \%$ \\
Samsung Galaxy S & S6 & S7 & S8 & S9 \\
Refurbishing scenario & $16 \%$ & $26 \%$ & $23 \%$ & $32 \%$ \\
Repair scenario & $7 \%$ & $10 \%$ & $9 \%$ & $13 \%$ \\
\hline
\end{tabular}

smartphones are replaced within one year (Wieser and Tröger 2017). Considering this age distribution, the allocation ratio results in 33\% for an iPhone not anymore in use and $22 \%$ for a Samsung Galaxy phone. Taking into account the market share of refurbished smartphones across the top 5 brands-40\% Apple and 60\% LG, Samsung, Sony and Huawei (Deloitte 2017) — the final average refurbishing allocation ratio is $27 \%$ for "one phone for smartphone refurbishing."

\subsubsection{Economic allocation for the semiconductor parts}

For the remanufacturing scenario, the market price for used components will also be used to derive the allocation value. Currently, the market price of used smartphone PCBs (including the input stream for the component remanufacturing) is mainly driven by material recycling (gold, copper, etc.). Since in the considered scenario only a few components are extracted from the $\mathrm{PCB}$, the material recycling and its profit are not significantly influenced, as gold and copper can still be recovered from the PCB afterwards.

The market price for remanufactured components is between 1 and $10 \%$ of the average original price. This price includes theoretically the costs for the used components (the input stream), the remanufacturing costs and the profit. The remanufacturing cost is about $0.70 €$ per component (Kopacek 2016), or about $2 \%$ of an average CPU (see also IHS). Expecting the profit will also be $1-2 \%$, the costs for used components and the remanufacturing allocation ratio are assumed to be $1 \%$ (per component).

\subsection{Definition second-use-time-parameter}

To allow the comparability of the results, and to scale the scenarios onto the chosen declared unit (one working smartphone for a use time of 2.5 years), second-use-timeparameters for each scenario are defined:

second use time parameter $=\frac{\text { avg second use time }}{\text { avg use time, linear scenario }}$

This parameter takes into account that the average second use time is shorter than in the first use phase of 2.5 years, as assumed in the declared unit (Wieser and Tröger 2017; Proske et al. 2016b). Depending on the used strategy (repair, refurbishing, remanufacturing), the product's second use time is shorter than the assumed average use time of 2.5 years for the first use phase, due to: 
Table 4 Second use time for refurbished smartphones, assumptions

\begin{tabular}{llllll}
\hline Input stream, age (years) & $0-1$ & $1-2$ & $2-3$ & $3-4$ & Avrg \\
\hline Distribution of age & $25 \%$ & $35 \%$ & $25 \%$ & $15 \%$ & \\
Second use time (years) & 2.5 & 2 & 1.7 & 1.2 & 1.93 \\
Total use time (years) & 3 & 3.5 & 4.2 & 4.7 & 3.73 \\
\hline
\end{tabular}

- Limitation by technical life time, requirements of current applications: The general smartphone's technical life time of 4.7 years (Ely 2014) represents the limit of the total use time. Accordingly, a refurbished phone of 3.5 years is expected to last a second life of maximum 1.2 years.

- Expectations and willingness of the customers: If a customer buys a 1-year-old refurbished smartphone, one could expect that the phone will be used for additional 2.5 years. Assuming the refurbished phone is already 3 years old, the second use time is likely to be 1.7 years.

To specify the parameter for refurbishment, the age of smartphones represented in the scenario's input stream is derived according to the smartphone replacement cycle survey data (Wieser and Tröger 2017). The smartphone's second use times are estimated considering factors as the technical life time (Table 4). The average smartphone's age in the input stream for refurbishment is 1.8 years, and the average second use time is 1.93 years. The second-use-timeparameter for refurbishing results in 0.77 .

For the repair scenario, the calculations are following the same logic as in the refurbishing scenario. The second use time is assumed to be maximum 2.5 years, if the smartphone needs repair right after buying it. The second use time decreases with the age of repaired phones (Table 5); the average second use time is 1.56 years. The second-use-timeparameter for repair results in 0.62 .

Within the remanufacturing scenario, all parts of the smartphone are new, except the remanufactured CPU,
Table 5 Second use time for repaired smartphones, assumptions

\begin{tabular}{llllll}
\hline Input stream, age (years) & $0-0.5$ & $0.5-1$ & $1-2$ & $>2$ & Avrg \\
\hline Distribution of age & $24 \%$ & $37 \%$ & $25 \%$ & $12 \%$ & \\
Second use time (years) & 2.25 & 1.75 & 1.25 & 0.5 & 1.56 \\
Total use time. (years) & 2.5 & 2.5 & 2.75 & 3.5 & 2.63 \\
\hline
\end{tabular}

RAM and the flash memory. It is assumed that the product's second use time can reach 2.5 years; therefore, the second-use-time-parameter for remanufacturing is 1 .

\section{Results}

\subsection{Environmental impact category}

According to the PCR "Methodology for environmental Life Cycle Assessment (LCA) of Information and Communication Technology, ETSI ES 203 199," the impact category for ICT goods, the Global Warming Potential, is taken into account. The GWP mainly highlights the energy consumption during the life cycle phases (ETSI 2015). Additional processes such as the disassembly and the transports for take back are well displayed in the GWP, but the avoidance of resource depletion, which is a main goal of CE, cannot be displayed. Therefore, the second impact category Abiotic Depletion Potential (ADP) is calculated and analysed in detail. Concluding, the environmental impact categories Global Warming Potential (GWP), Method IPCC 2013 GWP 100a and Abiotic Depletion Potential (ADP), Method EPD 2013 are selected to assess the environmental performance in this study.

\subsection{Environmental performance}

The result of the LCA (Fig. 6) shows the environmental impacts of the three end-of-use scenarios (repair, refurbishing and remanufacturing) compared with the linear

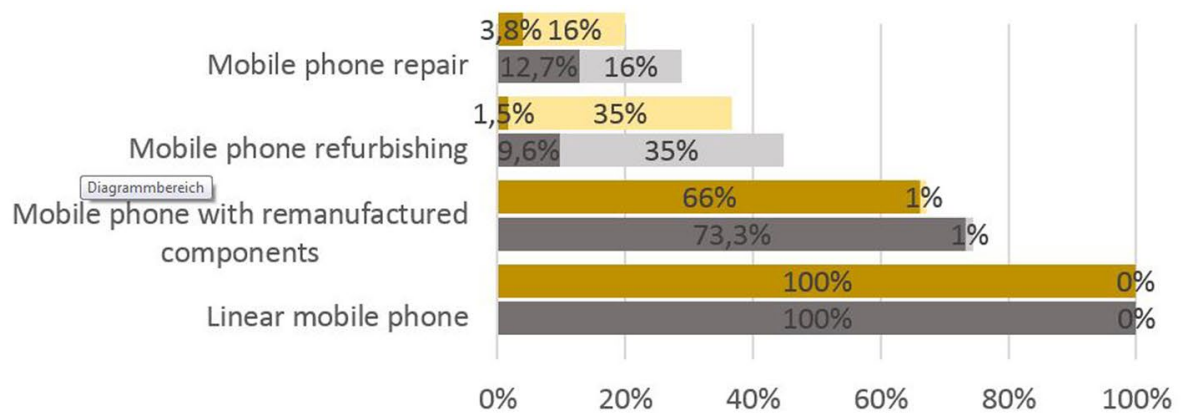

- Global Warming Potential (GWP), processes

Global Warming Potential (GWP), allocation burden

Abiotic Depletion Potential (ADP), processes

Abiotic Depletion Potential (ADP), allocation burden

Fig. 6 Environmental impacts of the individual scenarios, impact category GWP and ADP, considering a use time of 2.5 years 
smartphone scenario $\left(34.6 \mathrm{~kg} \mathrm{CO}_{2}\right.$ eq for materials, production and distribution). The results of all scenarios are aligned to the declared unit (2.5 years) by means of the second-use-time-parameters, in order to achieve comparability. The effort to keep phones and/or phone components in the loop and the allocation burden from the phone's first life are highlighted.

Taking into account the declared unit, the allocation approach and the second-use-time-parameter, the repair scenario shows the smallest environmental impact, whereas the remanufacturing scenario has the biggest impact. However, in comparison to the linear scenario, all three scenarios show a significant reduction of the GWP: $-25 \%$ for replacing a new smartphone by remanufacturing, $-55 \%$ by refurbishing and $-71 \%$ by repair. For the impact category ADP, an even higher reduction can be achieved. It is significant that the ADP allocation burden is more dominant than the GWP allocation burden. This is due to the resources that are already inside the smartphones and that are maintained through the different strategies. In contrast, the individual processes (Fig. 4) or
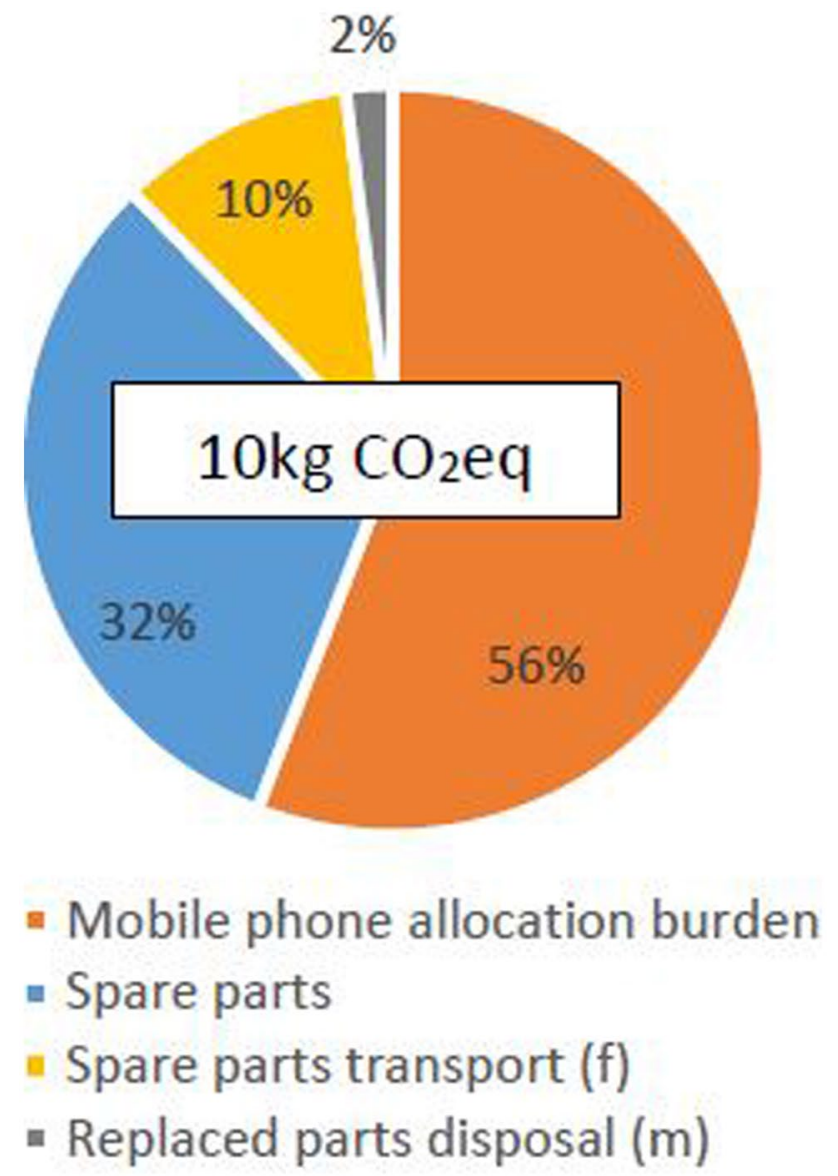

Fig. 7 Relative impacts due to smartphone repair, impact category GWP, considering a use time of 2.5 years transports tend to show higher GWP, and therefore, they are dominant.

The letters in the brackets in the description of Figs. 7, 8, and 9 refer to the overview graphic (Fig. 4), to identify recurring processes.

The share of the GWP for the two scenarios repair and refurbishing is shown in Figs. 7 and 8. The main GWP contributor for both scenarios with $56 \%$ and $78 \%$ is the allocation burden from the product's first life. This shows how important a chosen allocation method is.

In the repair scenario the repair process (h) itself is the second largest contributor with the materials and the production of the spare parts (32\%), followed by the transport (10\%). Also in the refurbishing scenario, all value-adding processes (testing, repairing, spare parts, data erasure) are second largest contributor with $14 \%$, followed by transports $(6.5 \%)$.

The distribution of the GWP in the remanufacturing scenario is shown in Fig. 9. This scenario is dominated by the (new) smartphone production and assembly ( $76 \%$ of total $\mathrm{kg}$ CO2eq), followed by the remanufacturing CPU, RAM and

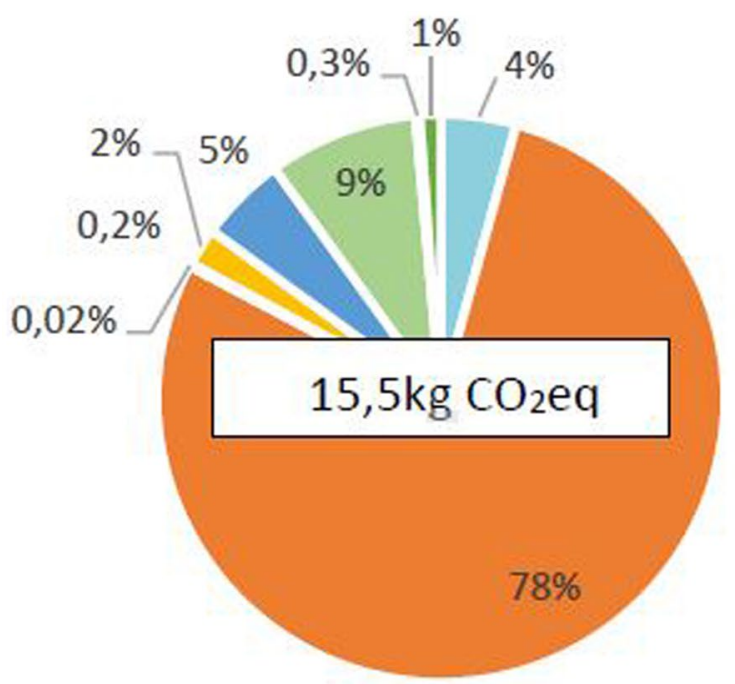

- Mobile phone collecting (a)

- Mobile phone allocation burden

- Mobile phone sorting (b)

- Mobile phone transport to refurbisher (d)

- Spare parts transport (f)

- Spare parts

- Mobile phone refurbishing (g)

- Replaced parts disposal (m)

Fig. 8 Relative impacts due to smartphone refurbishing, impact category GWP, considering a use time of 2.5 years 


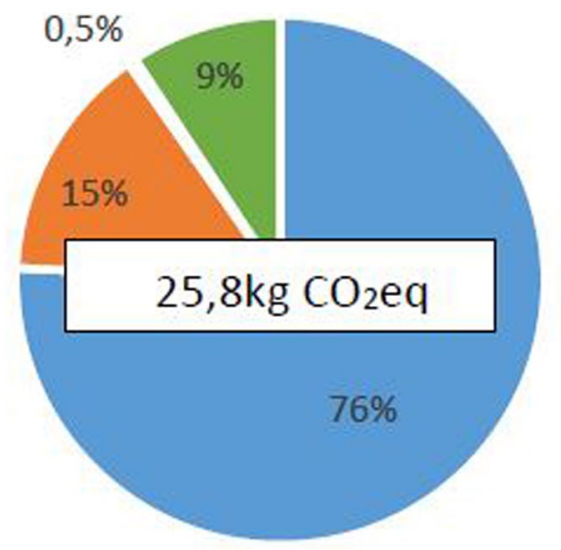

\section{- Mobile phone production and assembly (k) \\ - Remanufactured CPU, RAM- and flash memory \\ - Remanufactured components transport (j) \\ - Mobile phone distribution (I)}

Fig. 9 Relative impacts of a smartphone with remanufactured components, impact category GWP, considering a use time of 2.5 years

flash memory (15\%). Smartphone distribution from Asia back to Europe accounts for 9\% of the total GWP.

The component remanufacturing processes have been modelled and analysed in detail, and exemplarily highlighted for a remanufactured (smaller) Rockchip CPU in Fig. 10. Next to the process step "part de-soldering and remanufacturing" $(50 \%)$, the impacts are $45 \%$ due to "smart phone collecting" (Fig. 10). For remanufacturing bigger components, part de-soldering and remanufacturing become even more dominant, while the impact due to smartphone collecting stays the same (about $0.5 \mathrm{~kg}$ $\left.\mathrm{CO}_{2} \mathrm{eq}\right)$.

\section{Discussion and Conclusions}

\subsection{Environmental performance and influencing parameters}

Generally, it can be stated that the more parts and components can be reused; less resources will be needed for the production of new parts for the product's second life. For remanufacturing, the GWP of the production of new parts accounts for about $76 \%$ compared with just $32 \%$ for repair of the scenarios total GWP. The difference here is that in the remanufacturing scenario, about three quarters of the phone and its components need to be produced, whereas in the repair scenario, only some new spare parts for replacing broken ones are needed.

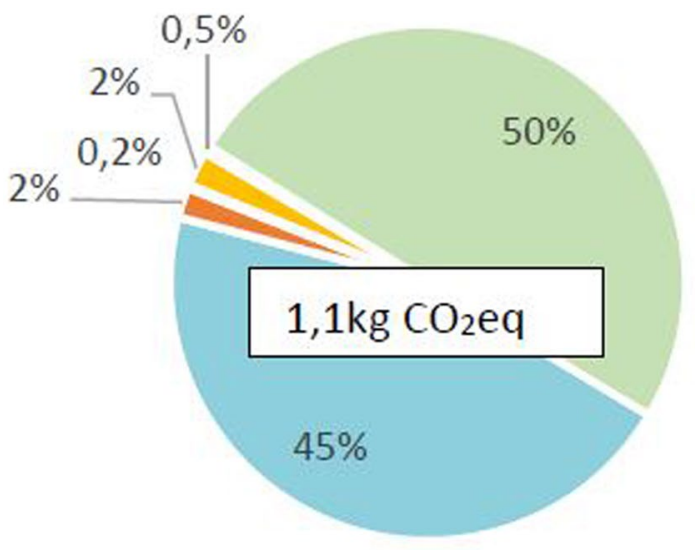

- Mobile phone collecting (a)

- Semiconductor allocation burden

- Mobile phone sorting (b)

- Mobile phone dismantling (c)

- PCB transport to remanufacturer (d)

- Part desoldering and remanufacturing (e)

Fig. 10 Relative impacts due to remanufacturing (a rather small) Rockchip CPU, impact category GWP

Transports show a lower impact compared with materials and production for new parts. For example, if the refurbishing site is near to the customer (e.g. in Europe, as assumed in the scenario), the GWP for transporting is about $6.5 \%$. Otherwise, in the remanufacturing scenario, for which it was assumed that the phones need to be transported from the manufacturer in Asia back to Europe (9\%), the transportation impact is higher $(11 \%$, including smartphone collecting and transports for component remanufacturing tasks). In the case of the repair scenario, the smartphone remains at the customer or in his immediate vicinity (repair shops etc. in a short distance of few $\mathrm{km}$ assumed), which means that there are only short transports for the complete smartphone but also transports for the spare part scenario, $10 \%$ of the scenarios total GWP.

The most relevant parameters influencing the environmental impact are as follows:

- Materials and production of the smartphone and new parts (in the range of $80 \%$ ): highly relevant for all three scenarios, influenced by design and production.

- Smartphone refurbishing or component remanufacturing steps (in the range of 10\%): influenced by the electricity grid mix.

- Transports (in the range of 10\%): influenced by type, distance, location of companies along the supply chain. 
Table 6 Repair scenarios for sensitivity analysis

\begin{tabular}{llll}
\hline Repair scenario & $\begin{array}{l}\text { Reparability suc- } \\
\text { cess factor }\end{array}$ & $\begin{array}{l}\text { Result, GWP } \\
(\mathrm{kg} \mathrm{CO} 2 \mathrm{eq})\end{array}$ & $\begin{array}{l}\text { Spare } \\
\text { parts } \% \\
\text { GWP }\end{array}$ \\
\hline Original & $75 \%$ & 10 & $32 \%$ \\
Moderate & $50 \%$ & 12.2 & $39 \%$ \\
Pessimistic & $33 \%$ & 15 & $45 \%$ \\
\hline
\end{tabular}

\subsection{Sensitivity analysis repair success factor}

In the repair scenario, the factor of successful repairs is assumed to be $75 \%$ (see also process i. Smartphone repair). As this is an optimistic assumption, two further scenarios were analysed ("moderate": factor 50\%-every second repair attempt is successful and "pessimistic": 33\%-every third repair attempt is successful) (see Table 6). It can be stated that the repair success factor has a high influence on the scenarios result, because spare parts needed account for $32 \%$ of the total GWP. In the two other scenarios (moderate and pessimistic), this even gets higher up to $45 \%$ as additional spare parts are needed for unsuccessful repair attempts. Still, even the pessimistic scenario shows a considerable reduction of GWP of $-57 \%$ compared with the linear reference scenario. Nevertheless, further research is needed for a solid and credible repair success factor and to determine the influencing factors like private repair attempts, professional repair shops etc.).

\subsection{Sensitivity analysis lighting and HVAC}

Some of the scenario's process steps were investigated and measured under lab conditions within the sustainablySMART project. Consequently, no data on lighting or heating, ventilation and air conditioning (HVAC) of an industrial site was collected. Instead, assumptions were included. In order to determine their influence on the results, a sensitivity analysis was carried out. Each scenario was modelled again, but with $a+200 \%$ increase in the energy overhead for lighting and HVAC. The scenario's total GWP increased by $+2 \%$ in the refurbishing scenario and $+17 \%$ in the remanufacturing scenario. The repair scenario remained nearly the same, and the energy overhead is not relevant. The analysis showed that lighting and HVAC does not necessarily make an important contribution for the repair and refurbishing scenario. For the remanufacturing scenario, the overhead energy could be relevant and more detailed data should be considered especially under a real production environment.

\subsection{System expansion modelling approach}

For the impact of the product's second life, the proposed allocation burden from the product's first life is about $56 \%$ for the repair scenario, and $78 \%$ for refurbishing scenario of their total GWP, but the burden is neglectable $<1 \%$ for the remanufacturing scenario. This result is strongly influenced by the chosen allocation method. The framework ISO 14044 suggests avoiding allocation and instead applying approaches such as system expansion, which means that the first and second product life are not assessed separately but together. The system expansion approach examined is the following: All life cycle phases starting from the materials and production of a phone in its first life, including the use phases from the phone's first and second life, up to the end-of-life after the second life are covered. The life cycle phase materials, production and distribution of the smartphone as well as the strategies after the first end-of-life phase are the same as in the allocation model. The duration of the use phases depends on the respective scenarios and order of use cycles and is the same as in the allocation model. For all use phases, an average energy consumption of 4 kWh/a (Proske et al. 2016a; Ercan and Kimfalk n. d.; Güvendik 2014) was assumed. For the end-of-life phase of the smartphone's second life, data based on literature (Proske et al. 2016a; Ercan and Kimfalk n. d.; Güvendik 2014) was used.

The results for 2.5 years of smartphone use are the following: repair scenario $41 \mathrm{~kg} \mathrm{CO}_{2} \mathrm{eq}$, remanufacturing scenario $35.2 \mathrm{~kg} \mathrm{CO}_{2} \mathrm{eq}$ and refurbishing scenario $30.3 \mathrm{~kg} \mathrm{CO}_{2} \mathrm{eq}$. These numbers show that the longer the smartphone's use (duration of second use phase after repair scenario < refurbishing scenario), the lower the environmental impact (repair scenario $>$ refurbishing scenario), unless the processes that enable a product's second life have a high environmental impact (remanufacturing scenario $>$ refurbishing scenario).

These results differ a lot from the ones derived from the applied allocation according economic value, where the repair scenario was the best option. The reason for this lays mainly within the system expansion where the environmental impacts from smartphones first and second life including their different use times are aggregated and then averaged based on the declared unit and not analysed separately. This means the result is not targeting/answering the goal of this study to guide decisions concerning CE-strategies of smartphones after its first life.

Although the different usage times of the use first and second use are taken into account, the quality of a smartphone cannot be taken into account. This quality reduces by the age of a smartphone, as e.g. the cover is scratched and need to be refurbished, or it also reduces when the smartphone gets broken. With the economic allocation model, this is considered using the ratio "residual market value, used or broken devices/original price." The consideration of the different quality together with the different usage times of the first and seconded use allow to decide which CE-strategy is most appropriate. 
To conclude according to the allocation model, if the decision is due and a smartphone is broken, the repair scenario should the first choice to enable an average usage time. In terms of the system expansion, a long smartphone usage is favourable as resources are used more efficiently. The refurbishing scenario has a much lower environmental impact than the repair scenario as the overall usage time (first and the second usage) is much longer (1.1 years). One could derive here that if a smartphone is broken, it should be repaired as first choice. As the usage time of a repaired device is still comparably short, the smartphone should go in a third use (cascading) where the phone is refurbished or remanufactured.

If a smartphone is used for about 2 years in the first use without repair needed, refurbishment and remanufacturing should be preferred, as the prolonging of the use time is than much longer than within the repair scenario.

Acknowledgements This research is part of the sustainablySMART project "Sustainable Smart Mobile Devices Lifecycles through Advanced Redesign, Reliability, and Reuse and Remanufacturing Technologies" which has received funding from the European Union's Horizon 2020 research and innovation programme under grant agreement No. 680604

\section{Funding Open Access funding provided by TU Wien (TUW)}

Open Access This article is licensed under a Creative Commons Attribution 4.0 International License, which permits use, sharing, adaptation, distribution and reproduction in any medium or format, as long as you give appropriate credit to the original author(s) and the source, provide a link to the Creative Commons licence, and indicate if changes were made. The images or other third party material in this article are included in the article's Creative Commons licence, unless indicated otherwise in a credit line to the material. If material is not included in the article's Creative Commons licence and your intended use is not permitted by statutory regulation or exceeds the permitted use, you will need to obtain permission directly from the copyright holder. To view a copy of this licence, visit http://creativecommons.org/licenses/by/4.0/.

\section{References}

Ciszewski P et al (2018) Remanufactured packaged semiconductors: technology validation. Report, sustainablySMART, https://www. sustainably-smart.eu/

Deloitte, 2017, Deloitte Global Mobile Consumer Survey 2017 -The Netherlands, https://www2.deloitte.com/content/dam/Deloitte/ nl/Documents/technology-mediatelecommunications/2017\% 20GMCS\%20Dutch\%20Edition.pdf , Accessed 14 May 2020

Ecoinvent (2020) Ecoinvent database v3.5, Swiss Centre for Life Cycle Inventories. Dübendorf, Switzerland. https://www.ecoinvent.org/ database/ecoinvent-36/ecoinvent-36.html. Last accessed June 2020

Ely C (2014) Consumer technology association-the life expectancy of electronics. https://www.cta.tech/News/Blog/Articles/2014/ September/The-Life-Expectancy-of-Electronics.aspx. Accessed 12 June 2019

Ercan E (2013) Global warming potential of a smartphone using Life Cycle Assessment methodology. Master of Science, Royal Institute of Technology, 2013 Stockholm
Ercan E, Kimfalk E (2016) Life Cycle Assessment of a smartphone, 4th International Conference on ICT for Sustainability (ICT4S 2016), Stockholm

ETSI (2015) Methodology for environmental Life Cycle Assessment (LCA) of information and communication technology (ICT) goods, networks and services, ETSI ES 203199 V1.3.1 (2015-02)

European Comission (2015) Closing the loop - An EU action plan for the Circular Economy. Brussels

Güvendik M (2014) From smartphone to futurephone-assessing the environmental impacts of different circular economy scenarios of a smartphone using LCA. Delft University of Technology and Leiden University

Janusz S et al (2016) Challenges of reuse and remanufacturing of modern chips in smart mobile devices. Electronics Goes Green Conference

Jkielty (2019) The smartphone upgrade cycle-visualized. https:// deviceatlas.com/blog/smartphone-upgrade-cycle-visualized. Accessed 16 July 2019

Kopacek B (2016) Intelligent disassembly of components from printed circuit boards to enable re-use and more efficient recovery of critical metals. IFAC Conf Paper, Elsevier, https://www.sciencedirect. com/science/article/pii/S2405896316325381

Kupka T (2018) Comparison of the environmental performance of standard printed circuit board production and embedded component packaging at AT\&S Leoben, Diploma Thesis TU Wien

Mishra V (2018) Growth of refurbished smartphones in emerging markets. https://www.counterpointresearch.com/increasing-growthemerging-markets-refurbished-smartphone-segment/. Accessed 16 July 2019

Pamminger R et al (2019) Digital Voice recorder with reuse components: technology validation. Report, sustainablySMART, https:// www.sustainably-smart.eu/

PRé (2020) PRé Consultants. SimaPro database manual-methods library: http://www.pre-sustainability.com/download/ DatabaseManualMethods.pdf. Last accessed June 2020

premium concepts GmbH (2013) Infografik über kaputte Smartphones. https://handyreparatur123.de/infografik-ueber-kaputtesmartphones/. Accessed 06 June 2019

Proske M et al (2016a) Life Cycle Assessment of the Fairphone 2. Final Report, Fraunhofer IZM, Berlin, https://www.fairphone.com/wpcontent/uploads/2016/11/Fairphone_2_LCA_Final_20161122.pdf

Proske M et al (2016b) Obsolescence of electronics-the example of smartphones. Electronics Goes Green Conference 2016, Berlin, http://publica.fraunhofer.de/documents/N-467567.html

Refurbed GmbH, 2019, Interview with Kilian Kaminski (CEO), 07 March 2019 and https://www.refurbed.at, Accessed 08 March 2019

Skerlos S et al (2003) Economic and environmental characteristics of global cellular telephone remanufacturing. IEEE International Symposium on Electronics and the Environment, https:// www.semanticscholar.org/paper/Economic-and-environmentalcharacteristics-of-Skerlos-Morrow/b6d0a354b6016e7f2182399f3 a50da5ebef87e6b

Statista (2016) Welche Arten von Schäden hatten Sie bisher schon an Ihren Smartphones? https://de.statista.com/statistik/daten/ studie/663298/umfrage/umfrage-zu-schaeden-an-smartphones-indeutschland/. Accessed 16 July 2018

Wieser H, Tröger N (2017) Exploring the inner loops of the circular economy: Replacement, repair, and re-use of mobile phones in Austria. Elsevier, https://www.sciencedirect.com/science/article/ abs/pii/S0959652617327798

Publisher's Note Springer Nature remains neutral with regard to jurisdictional claims in published maps and institutional affiliations. 